\title{
Diamond Photovoltaic Cell Development
}

\author{
N. B. Rodionov, V. N. Amosov, S. A. Meshchaninov, and A. F. Pal
}

\begin{abstract}
A synthetic diamond based heterostructure (p-i-structure) is examined as a photovoltaic cell. P-type diamond heavily doped with boron was used as a substrate. A CVD IIa-type diamond film of $50 \mathrm{mkm}$ thickness was deposited on the substrate.

A technology of semi-transparent entrance contact fabrication was developed. A current-voltage characteristic (I-V) of the wide band gap diamond photovoltaic cell were measured. For the case of UV irradiation the comparison of I-V for ordinary and semi-transparent contacts was performed. Experimental data allowed estimating of alpha, x-ray and UV radiation conversion efficiency to be in the range $5 \div 7 \%$.
\end{abstract}

Index Terms-Boron doped diamond, CVD diamond, diamond heterostructures, photovoltaic cell, semi-transparent contact.

\section{INTRODUCTION}

Currently there is a lack of the compact autonomous power generators capable to operate for a long time. UV sources or radioactive isotopes in couple with photovoltaic cells converting photon energy into electric current are promising candidates for power sources.

A number of UV and radioactive decay energy conversion methods have been developed [1]. In case of power needs up to hundreds of watts the radionuclide thermoelectric current generators (RTGs) are used [1] for a variety of applications like automatic meteorology stations, lighthouses, satellites.

Sr-90 and Pu-238 (for high power supplies) are major fuel for RTGs. New opportunities of nuclear power source characteristics improvement have appeared after photovoltaic converters based on wide bang gap semiconductors like doped synthetic diamond [2]-[4] were proposed. To convert radiation energy into electric current photovoltaic effect of diamond can be used [5], [6]. A semiconductor structure containing built-in electric field regions where separation of photo generated charge carriers occur is the basis of such devices.

The advantages of synthetic diamond based devices are radiation hardness, high operating temperature (up to $150 \mathrm{C}$ ), high thermal conductivity, chemical inertness.

Simple and compact power source with ability to work during tens of years can be created on the basis of these

Manuscript received November 14, 2014; revised June 14, 2015. This work has been carried out with financial support of the Ministry of education and science under the AGREEMENT No. 14.579.21.0030 for A SUBSIDY in the framework of the Federal target program "Research and development on priority directions of development of scientific-technological complex of Russia for 2014-2020". Unique scientific research identifier RFMEFI57914X0030.

All authors are with the State Research Center of Russian Federation Troitsk Institute for Innovation \& Fusion Research, Troitsk, Moscow, Russia, 142190 (e-mail: rodionovnb@gmail.com). elements.

In work [7] the diamond detector's sensitivity of different types of radiation (UV, x-rays, charged particles, fast neutrons) was determined for steady and pulse irradiation regimes. The current-voltage characteristics were obtained. Those characteristics have a quasi-diode shape with clear photovoltaic shift of 1.6 Volts. This effect is due to boron substrate and deposited CVD diamond film interface. Every interface on the pure diamond surface creates contact voltage, while high electric charge mobility provides low loses of charge collection. This fact can be utilized in photovoltaic converters of different types.

The goal of present work is to develop synthetic diamond based photovoltaic converters. Thin film (p-i) structure was considered as photovoltaic converter and its photovoltaic characteristics were measured. A technology of semitransparent contacts fabrication was developed to ensure effective short-wave radiation entrance into converter's volume.

\section{DISCUSSED PROBLEMS}

A (p-i)-structure for photovoltaic cell is a substrate with deposited CVD diamond film. The substrate is HTHP IIb-type diamond highly doped with boron substrate (p-type) and has very good hole conductivity. Substrate dimensions were $4 \times 4 \times 0.5 \mathrm{~mm}^{3}$. Nitrogen concentration was less than 1 ppm. Boron concentration was $100 \mathrm{ppm}$. CVD IIa-type diamond film with $50 \mathrm{mkm}$ thickness was deposited on the substrate. On the external CVD diamond surface of ordinary or semi-transparent metal contact was deposited. On the substrate bottom side ordinary metal contact was deposited. Photovoltaic converter based on (pi)-structure is shown in Fig. 1. Arrows indicate the direction of incident radiation. The working volume of the photovoltaic cell is a diamond layer of type IIa, adjacent to the substrate. Boron-doped diamond material has sufficient hole conductivity and diffusion velocity magnitudes in the material of i-type to created EMF photovoltaic cell.

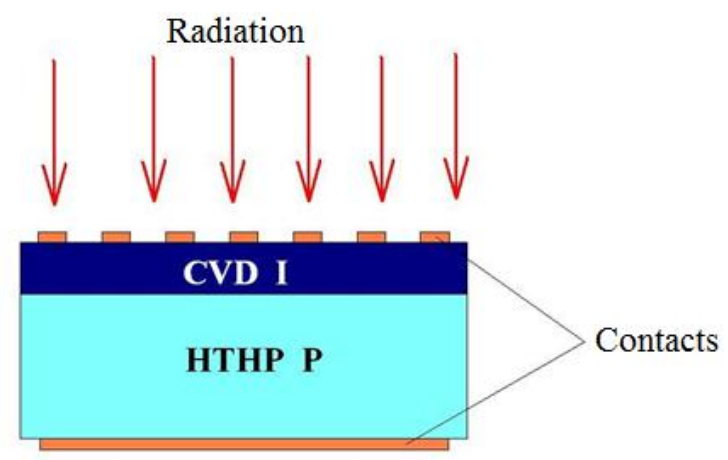

Fig. 1. The photovoltaic cell based on p-i-structure: p-type HTHP diamond substrate with deposited CVD IIa-type diamond film of $50 \mathrm{mkm}$ thickness. 


\section{MANUfACtURING (P-I)-STRUCTURE}

\section{A. Preparation of the Substrate}

HTHP diamond crystals were annealed in a vacuum high-temperature furnace RD-G WEBB-117. An influence of vacuum annealing on the HTHP diamond conduction was investigated. The main parameters of the substrate under consideration are the absorption spectrum under UV and IR radiation and the conductivity of the substrate at room temperature. The absorption spectra and conductivity were measured for a range of annealing temperatures from $10000 \mathrm{C}$ to $16000 \mathrm{C}$ with $1000 \mathrm{C}$ increments. Also cathodoluminescence and $\mathrm{X}$-ray luminescence intensities were measured. We investigated characteristics of the two crystals samples: $\mathrm{Br} 40, \mathrm{Br} 03$ (according to the classification of FSUE "SRC RF TRINITY"). Cathodoluminescence becomes less luminous with increasing annealing temperature. Measurements of X-ray luminescence also showed that annealing decreases contrast and brightness of the luminescence. This means that annealing decreases the concentration of crystal's defects. After each annealing chemical cleaning of the samples has being carried out because the annealing leads to partial graphitization of the crystal surface.

The electrical resistance of the crystal was determined by the tangent to the I-V for "large" (not near zero) voltages. I-Vs were measured in the bound regime of operation of the electrometer without radiation in the temperature range $1100^{\circ} \mathrm{C}-1500^{\circ} \mathrm{C}$. A significant change of $\mathrm{Br} 03$ resistance started after annealing at $1300^{\circ} \mathrm{C}$ and decreased with the subsequent annealing steps by about 10 times. The resistance of $\mathrm{Br} 40$ decreases monotonically throughout all stages of annealing, except the last one at $1500^{\circ} \mathrm{S}$, reaching a value of $50 \mathrm{ohms}$, which is less than the initial value by 2.3 times.

\section{B. Deposition CVD Diamond}

After the substrate has been annealed and good conductivity has been achieved the p-i-structure was fabricated: homoepitaxial CVD layer of ultimate purity IIa-type diamond with 50 microns thickness was deposited on the substrate. On the back side of the sample a solid gold electrical contact of $30 \mathrm{~nm}$ thickness was deposited. On the front side (on the CVD film) semitransparent contact was deposited. It has $3 \mathrm{~mm}$ width and consisted of strips with 20 microns widths and 50 microns distance between them. The process of deposition of the epitaxial diamond film doped with boron requires careful preparation of the original substrates surface. Cathode-luminescence pattern obtained by scanning of the substrates with an electronic microscope showed that there were zones with an increased defectiveness in the samples, which correspond to the zonal inhomogeneity of substrates and appeared as bright cross-shape bands i.e. the substrates, had a blok structure. Such structure decrease the quality of homoepitaxial films and imperfectness crystal structure of those films reduce the conversion efficiency of ionizing radiation into electric current.

\section{Manufacturing of Photovoltaic Cell}

To obtain UV semitransparent gold contacts on diamond crystal magnetron deposition facility DESK V was used. First continuous layer of metal was deposited on the crystal. Then, special mask was laid on the crystal surface. After that, crystal with mask was placed in an ion cleaning installation, where uncovered regions of metal were removed by ion beam. Semitransparent gold contact profile, measured on an atomic force microscope (AFM), is shown in Fig. 2.

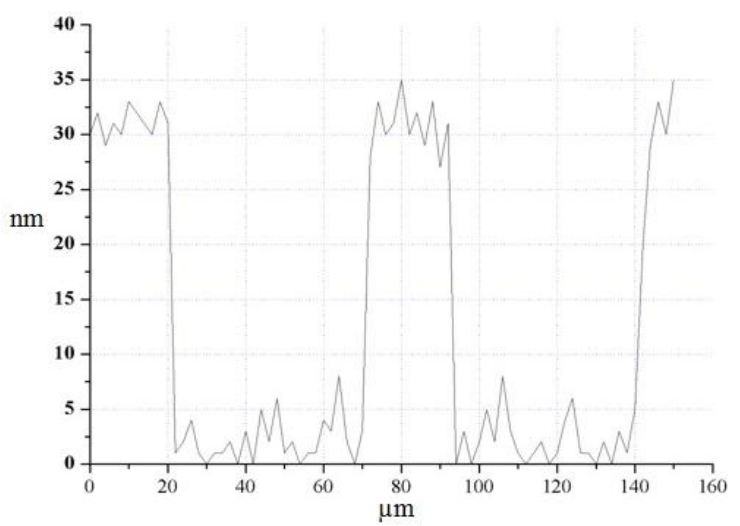

Fig. 2. Semitransparent gold contact profile, measured on an atomic force microscope (AFM).

Several types of masks were considered for deposition process. Masks were fabricated from copper by laser cutting.

$\mathrm{P}$-i-structure with gold contacts has been installed in the detector housing by spring contacts. The face contact had shape of a diaphragm with entrance aperture of $2 \mathrm{~mm}$ in diameter. The converter has been tested under different conditions of exposure on purpose of conversion of UV, EUV and $\mathrm{X}$-ray radiation into electricity.

Standard spectrometry electronic consisting of CanBerra 2001A charges sensitive preamplifier, shape amplifier BUI-14P, ADC "Parsek" has been used.

\section{EXPERIMENTAL EXAMPLES AND ANALYSIS}

The characteristics of the $\mathrm{p}$-i-structure based photovoltaic cell have been tested. Tests of charge collection efficiency of $\mathrm{p}$-i-structure irradiated by alpha particles showed that the charge collection efficiency exceed $90 \%$ and almost reached $100 \%$ when calculated electric field in the film $>0.5 \mathrm{~V} / \mathrm{m}$. $5.5 \mathrm{MeV}$ alpha particles peak was measured with an energy resolution of $2 \%$. The alpha particles flux $5 \times 10^{2}$ was equal to $0.44 \times 10^{-9}$ watts of absorbed energy. Measured I-V are shown in Fig. 3. 40 pA current was registered when zero bias voltage was applied (short circuit current, Isc). Taking into account the $0.62 \mathrm{~V}$ photovoltaic voltage shift (open-circuit voltage, Uoc), the converter efficiency is concluded to be equal to $5.4 \%$.

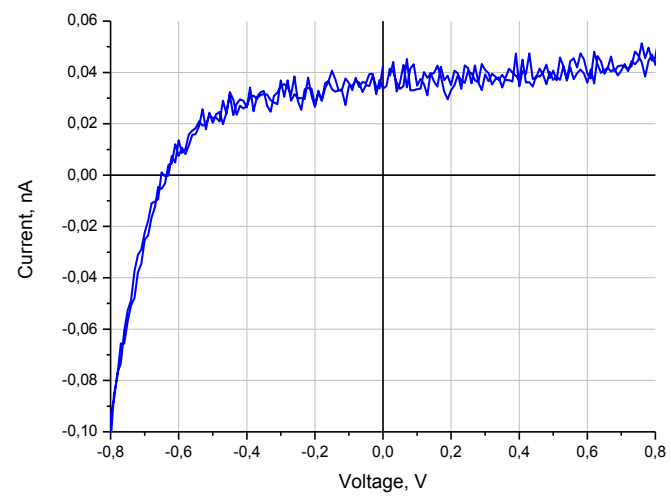

Fig. 3. I-V of p-i structure under irradiation by alpha particles. 
The current-voltage characteristic of the sample under X-ray irradiation with a $1 \mathrm{Rad} / \mathrm{sec}$ dose rate is shown in Fig. 4. The negative branch of the I-V has rapidly growing character due to current injection amplifying and depends linearly on the magnitude of the electric field on the boundary of injecting contacts between the epitaxial film and the boron substrate. Taking into account the dose $\sim 1 \mathrm{Rad} / \mathrm{sec}, 1.4 \mathrm{~V}$ shift photovoltaic and current at zero bias is $1.8 \mathrm{nA}$ (Isc) evaluated the efficiency which in this case was equal to $6 \%$.

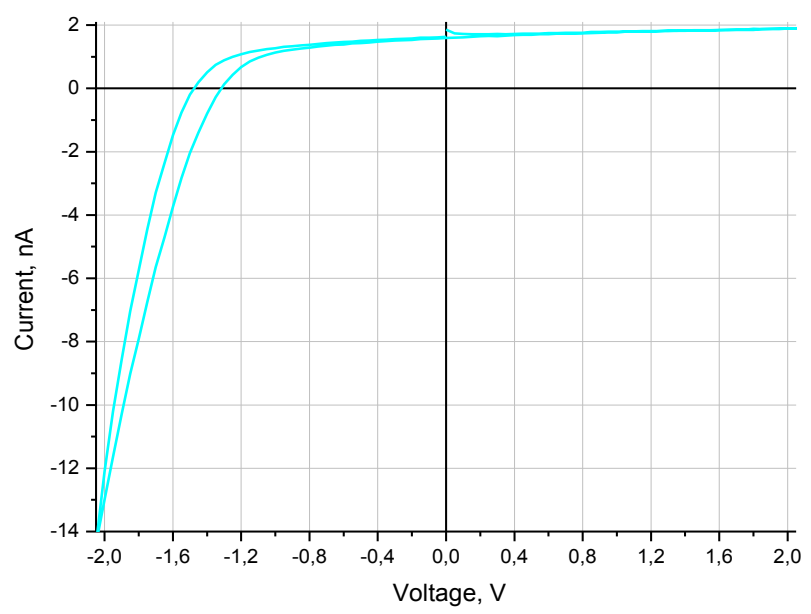

Fig. 4. I-V of the diamond structure under X-ray irradiation with a dose rate of the order of $1 \mathrm{Rad} / \mathrm{sec}$.

The I-V of the inverter irradiated by UV deuterium lamp is shown in Fig. 5. Two types of contacts were explored: ordinary (solid curve) and semi-transparent (dashed curve). The curves have a clear photovoltaic shift which is equal to $-1.2 \mathrm{~V}$. Semi-transparent contact allows to increase efficiency of the photovoltaic cell almost by factor of two. In the last case efficiency didn't exceed $7 \%$.

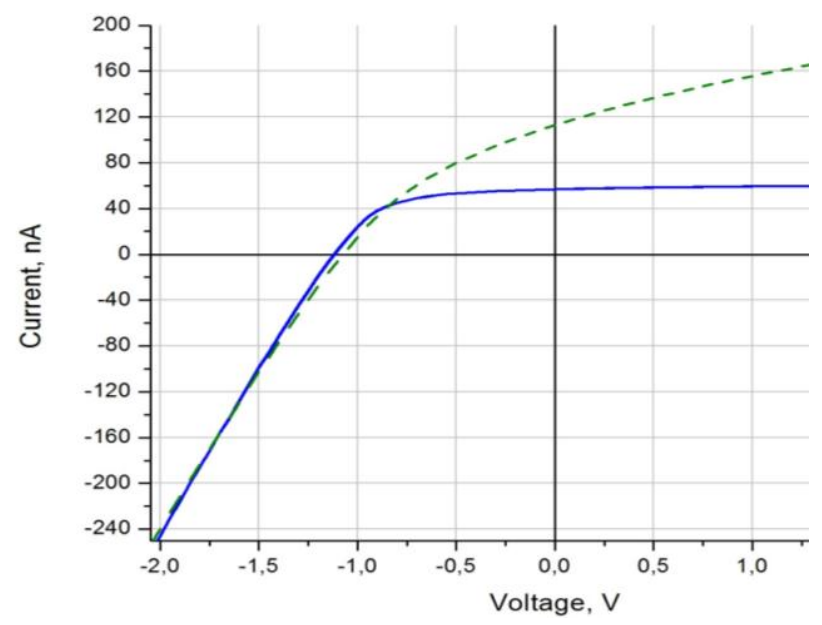

Fig. 5. I-V under deuterium lamp UV irradiation (dashed curve semi-transparent contact; solid curve - a ordinary contact).

Fig. 6 demonstrates the image of the crystal with semitransparent contact which was obtained on the microscope in transmitted light.

Thus, the created diamond structure was shown to be able to operate as the converter of radiation into electric current. The conversion efficiency of ionizing radiation into electric current depends on the quality of homoepitaxial films. Defects (imperfect) crystal structure of those films reduce the average lifetime of the charge carriers in the film. Further improvement of performance is possible with improvement of the quality of the substrate used, and the surface preparation quality during film deposition process. Photovoltaic converter with large area and high effectiveness can be built by connecting described photovoltaic cells in parallel circuit."

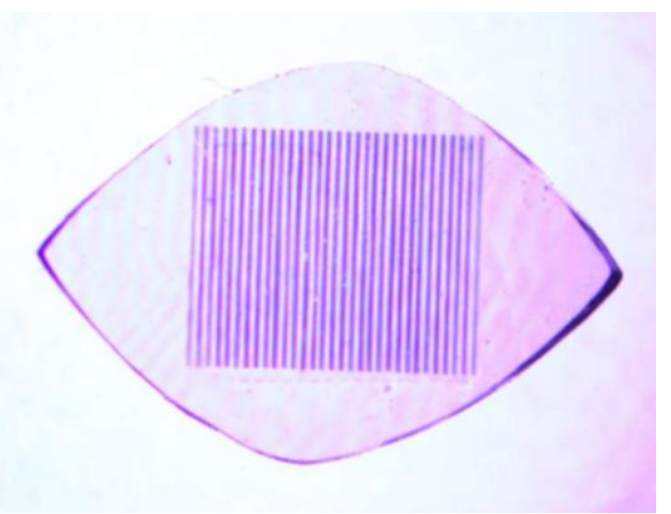

Fig. 6. Image of crystal with semitransparent contact.

\section{CONCLUSION}

Diamond p-i structure has demonstrated the ability to operate as a converter of radiation into electricity. The technology of manufacturing of semi-transparent windows for input photovoltaic cell has been developed. Diamond photovoltaic cell with ordinary and semi-transparent entrance windows have been created. Testing of converter under UV, alpha-and X-ray radiation has been performed. The converter efficiency was estimated to be in the range of $5 \% \div 7 \%$.

\section{REFERENCES}

[1] G. A. Cherevatenko, E. D. Chistov, and V. M. Kodukov, Radionuclide Sources in Radiation Technology, Energoatomizdat, 1989.

[2] M. Prelas, G. Popovichi, S. Khasawinah, and J. Sung, Wide Band Gap Materials, Minsk, p. 463, 1994.

[3] G. Popovichi, A. Melnikov, V. V. Varichenko, S. Khasawinah, T. Sung, M. Prelas, and R. G. Wilson, "Diamond ultraviolet photovoltaic cell obtained by lithium and boron doping," J. of Appl. Phys., vol. 81, no. 5, pp. 2429-2431, 1997.

[4] V. I. Polyakov, A. I. Rukovishnikov, N. M. Rossukanyi, V. G. Ralchenko, F. Spaziani, and G. Conte, "Photoconductive and photovoltaic properties of CVD diamond films," Diamond \& Related Materials, vol. 14, pp. 594-597, 2005.

[5] F. Spaziani, M. C. Rossi, S. Salvatori, and G. Conte, "Optimized spectral collection efficiency obtained in diamond-based ultraviolet detectors using a three-electrode structure," Appl. Phys. Lett., vol. 82, no. 21 , p. 3785,2003

[6] D. M. Trucchi, E. Cappelli, N. Lisi, and P. Ascarelli, "Feasibility of CVD diamond radiation energy conversion devices," Diamond \& Related Materials, vol. 15, pp. 1980-1985, 2006.

[7] V. N. Amosov, E. A. Azizov, V. D. Blank, N. M. Gvozdeva, N. V. Kornilov, A. V. Krasilnikov, M. S. Kuznetsov, S. A. Meshchaninov, S. A. Nosukhin, N. B. Rodionov, and S. A. Terent'ev, "Development of ionizing radiation detectors based on synthetic diamond material for the nuclear power industry," Instruments and Experimental Techniques, vol. 53, no. 2, pp. 196-203, 2010.

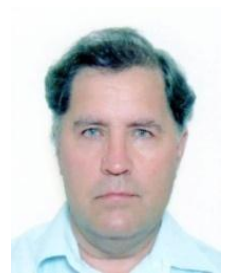

Nikolay Rodionov was born in the USSR on Jan. 15 1952. He graduated from Moscow Engineering Physical Institute in 1975. He received his Ph.D degree of physics and mathematics in the field of radio physics and quantum electronics, from P.N. Lebedev Physical Institute of the USSR Academy of Sciences, Moscow, Russia in 1979. He received his doctor degree of physics and mathematics, in the field of 
laser physics, Moscow State University, Moscow, Russia in 2003. His main science activities include laser physics, hydrodynamic, IRSH of thermonuclear plasma tokamaks.

He worked as a junior researcher, researcher, senior researcher, lead researcher in SRC RF TRINITI since 1978. At present he is the director of the Scientific Educational Center: The Center of Radiation Resistive Diamond Nanoelectronics and Innovations of SRC RF TRINITI.

Dr. Nikolay Rodionov is the author of more than one hundred scientific publications and reports, he is the leader of a number of scientific projects, and a member of science council of Corporation SRC RF TRINITI.

Vladimir Amosov was born in the USSR on Nov. 29, 1957. He graduated from Moscow Institute of Physics and Technology in 1981. His main science research includes plasma physics, neutron diagnostics.

He worked as a junior researcher, the head of scientific group, a senior researcher in SRC RF TRINIT, since 1981.

Mr. Amosov Vladimir is the author of more than fifty scientific publications and reports.

Sergei Meshchaninov was born in the USSR on Jun. 29, 1981. He graduated from Moscow Engineering Physical Institute in 2005. His main science research includes semiconductor detectors, neutron diagnostic.

He worked as engineer, researcher in SRC RF TRINITI, since 2009.

Mr. Sergey Meshchaninov is the author more than fifteen scientific publications and reports.
Alexander Pal was born in the USSR on Nov. 9, 1941. He graduated from the Physics Department of Moscow State University in 1966. He received his $\mathrm{PhD}$ degree in physics of plasma from Institute of Nuclear Physics of Moscow State University in 1978. He got the doctor of science degree of plasma physics from I.V. Kurchatov Institute in 1993. His main science research includes low-temperature plasma, gas discharge, laser physics, dusty plasma, plasma technologies, plasma assisted CVD.

He worked as a junior researcher and leading engineer in the Institute of Nuclear Physics of Moscow State University in 1969-1983, then worked as a senior researcher, leading researcher, the head of laboratory, and the head of department in SRC RF TRINITI in 1983-2007. Then he has been working as a leading researcher in Institute of Nuclear Physics of Moscow State University since 2007.

Dr. Alexander Pal is the author of more than 100 scientific publications and patents.

Alexander Trapeznikov was born in the USSR on Aug. 25, 1979. He graduated from Moscow Institute of Physics and Technology in 2003. He was a post graduate student in the field of plasma physics, at Troitsk Institute for Innovation and Fusion Research, Moscow, Russia, 2003-2007. His main science research includes plasma physics, solid state physics, neutron diagnostic.

He worked as an engineer and researcher in SRC RF TRINITI, since 2003.

Mr. Alexander Trapeznikov is the author of more than ten scientific publications and reports. 